\title{
CONSIDERACIONES ECONÓMICAS SOBRE LOS OBJETOS DE CONCHA EN TIERRAS BAJAS DEL ÁREA MAYA
}

\section{ECONOMIC CONSIDERATIONS ON SHELL OBJECTS IN THE LOWLANDS OF THE MAYAN AREA}

\author{
Rafael Cobos ${ }^{1}$ \\ RACHCOBOS@aol.com / Orcid https://0000-0001-6409-7348
}

\section{RESUMEN}

Una perspectiva netamente económica se sigue en este trabajo para analizar la producción de objetos de concha en sitios de las Tierras Bajas del área Maya durante los períodos Clásico Tardío y Clásico Terminal. Primero analizamos seis diferentes tipos de abastecimiento de materia prima que fue trabajada para producir distintos tipos de objetos utilitarios y ornamentales. Posteriormente nos enfocamos a la forma en la cual se organizó la producción, en otras palabras, si fue de manera independiente en unidades domésticas tanto de la elite como de miembros no pertenecientes a la elite, o bien, esta producción se realizó de forma sistemática en talleres.

Palabras clave: Conchas; Maya; Arqueologia.

\footnotetext{
${ }^{1}$ Profesor-investigador, Universidad Autónoma de Yucatán, Mérida, México.
} 


\title{
Clio Arqueológica 2021, V36N2, p.149-176, COBOS;

\begin{abstract}
An economic perspective is followed in this article to analyze the production of shell objects at different settlements in the Maya lowlands during the Late Classic and Terminal Classic periods. The analysis of six different forms of procurement of raw material, which was worked to produce different types of utilitarian and ornamental objects, is presented in the first part of this contribution. The organization of production is the goal of the second part and it is questioned whether this was done independently in household units of both elite and non-elite members, or whether this production was done -systematically in workshops
\end{abstract}

Key words: Shell; Maya; Arqueology.

\section{CONTEXTO DE LA INVESTIGRACIÓN}

Los estudios de conchas arqueológicas en las Tierras Bajas del área Maya nos

señalan hoy día las distintas regiones marino-costeras y de agua dulce desde las cuales se obtuvieron los materiales para elaborarlos; también nos presentan y describen la gran variedad de piezas ornamentales, utilitarias y votivas que se utilizaron en diferentes contextos en esos sitios; nos muestran los cuantiosos fragmentos que evidencian las huellas de manufactura de los objetos (ANDREWS IV, 1969; VOKES Y VOKES, 1983; ABBOTT, 1996; VELÁZQUEZ Y LOWE, 2007; COBOS, 2010, 2014; HERNÁNDEZ et al., 2014; MELGAR, 2016; GÓMEZ Y PANTOJA, 2018).

Además, estudios experimentales replican hoy día, empleando análisis tecnológicos, los pasos factibles que se siguieron, así como las posibles herramientas que se utilizaron en la obtención de piezas utilitarias, ornamentales y

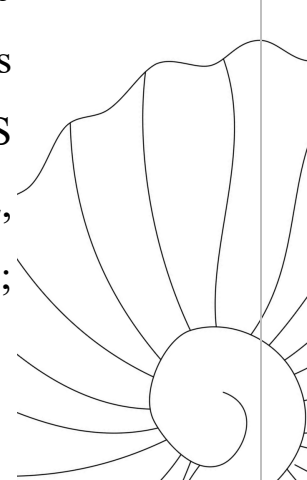


votivas (VELÁZQUEZ Y LOWE, 2007; VELÁZQUEZ et al., 2013; MELGAR Y DOMÍNGUEZ, 2017; REYES, 2018; COTOM, 2019; CASTILLO, 2020). Sin embargo, el estudio en sí mismo de la producción, distribución y consumo de objetos de concha es un campo que aún debe ser estudiado sistemáticamente y utilizando un enfoque predominantemente económico (ver por ejemplo -FEINMAN Y NICHOLAS, 1993; 1995; 2004).

Los estudios económicos de los objetos de concha radican en explicar en donde se obtuvo la materia prima y los lugares en los cuales se manufacturaron los distintos objetos (producción); cómo se repartieron esos objetos tanto regional como localmente (distribución); quienes obtuvieron los objetos para utilizarlos en distintos contextos a través del tiempo (consumo). En este trabajo solamente analizamos dos de los aspectos económicos relacionados con la producción de objetos concha. Estos aspectos incluyen (a) la forma en la cual se obtuvo la materia prima y (b) la forma en la cual se organizó la producción, en otras palabras, si fue de manera independiente en unidades domésticas tanto de la elite como de miembros no pertenecientes a la elite, o bien, fue organizada de forma sistemática en talleres. A continuación, explicamos.

\section{¿CÓMO SE OBTUVO LA MATERIA PRIMA?}

Como primer punto a considerar en el proceso de producción es la identificación del litoral desde el cual se obtuvieron conchas marinas para ser transportadas por varios kilómetros a los sitios del interior. Una mirada a la Tabla 1 revela que

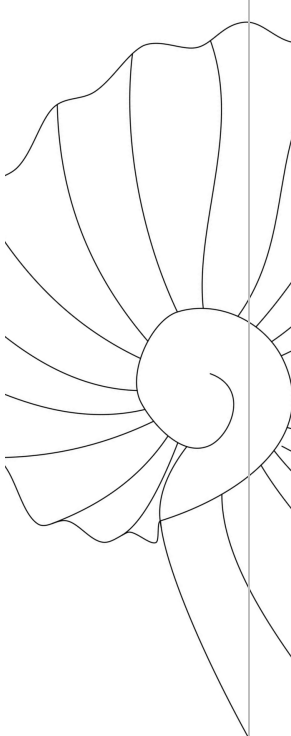




\section{Clio Arqueológica 2021, V36N2, p.149-176, COBOS;

asentamientos tanto de las Tierras Bajas Mayas del norte como del sur prefirieron abastecerse de conchas nativas de las regiones del Golfo de México y Mar Caribe y, en menor grado, de especies nativas del Océano Pacífico. Esta preferencia entre los dos patrones de abastecimiento sugiere que los sitios de Tierras Bajas Maya aprovecharon su posición geográfica con respecto a la cercanía de los litorales del Golfo de México y Mar Caribe (Figura 1).

Los dos patrones de obtención de materia prima en las Tierras Bajas Mayas podrían explicarse empleando la palabra 'comercio', sin embargo, esta palabra puede resultar engañosa. El hecho de encontrar piezas de concha propias del litoral marino en algún sitio del interior no puede utilizarse para asumir y afirmar categóricamente que las piezas llegaron únicamente por 'comercio', es decir, si 'comercio' se define o entiende como intercambio de mercado (FEINMAN Y GARRATY, 2010: 169-172; HIRTH, 2016: 60-61; ver también FEINMAN Y NICHOLAS, 1993; 1995; 2004). En otras palabras, las piezas de concha también pudieron haber llegado por alguna de las siguientes maneras: (1) artesanos solicitaron por encargo la materia prima para que les fuese enviada hasta sus respectivas comunidades (RENFREW, 1975: 41-43, Figura 10-1), (2) artesanos de las distintas comunidades fueron hasta el litoral marino o fuentes de agua dulce a obtener la materia prima (por ejemplo, el caso de la obtención de mica de Teotihuacan [MANZANILLA et al., 2017]; ver también RENFREW, 1975: 4143, Figura 10-1), (3) artesanos obtuvieron la materia prima de abastecedores que la pudieron haber transportado hasta un punto intermedio entre la comunidad y la

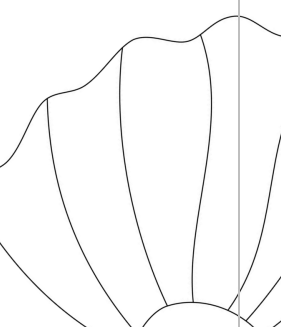


costa marina (RENFREW, 1975: 43-44, Figura 10-3), (4) por medio de tributo o impuesto (BERDAN et al., 2003; Smith 2004), (5) por reciprocidad balanceada y/o generalizada (SMITH, 2004), (6) por robo a individuos quienes transportaban la materia prima (SMITH, 2004).

El intercambio de mercado y las seis formas de abastecimiento de la materia prima arriba mencionadas pudieron haber operado al mismo tiempo en un mismo sitio y/o región, o bien, quizás solamente una de ellas fue la predominante. Por lo tanto, la pregunta que surge es la siguiente: ¿cómo podemos reconocer arqueológicamente cada una de esas formas de abastecimiento? Por ahora, y con base en la evidencia arqueológica, podemos responder a esta pregunta parcialmente y de varias maneras.

Una de las primeras formas en la que podemos responder a la pregunta es cuando consideramos a los artesanos quienes solicitaron por encargo la materia prima que llegó hasta sus respectivas comunidades. De hecho, esto resulta evidente considerando la relación entre Chichén Itzá y su puerto Isla Cerritos ya que, una comparación entre las especies de moluscos marinos fechadas para el período Clásico Terminal entre la ciudad localizada en el interior de Yucatán y su puerto en la costa del Golfo de México, revela que se trata de las mismas especies marinas (COBOS, 1989; 2014). Por lo tanto, podemos asumir que artesanos de Chichén Itzá se beneficiaron de la obtención de materia prima por parte de los residentes permanentes del puerto que Chichén Itzá estableció en el litoral marino

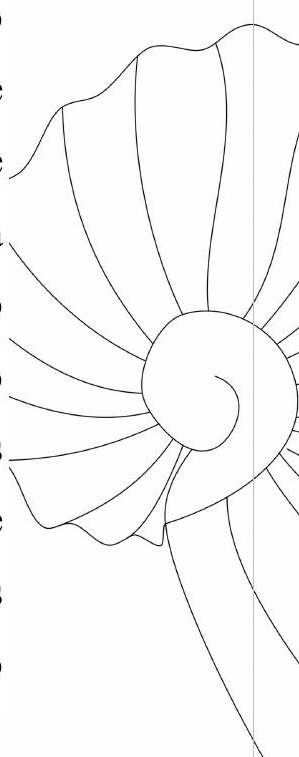


y sin la participación de terceras personas. La evidencia de conchas arqueológicas halladas en Isla Cerritos y Chichén Itzá sugiere que bien pudieron haberse organizado grupos de individuos para recolectar distintas conchas y trasladarlas y/o enviarlas directamente hasta Chichén Itzá.

Sin embargo, no todos los sitios de las Tierras Bajas Mayas tuvieron puertos marítimos en el litoral y esto hace que consideremos de nueva cuenta el intercambio de mercado y las seis formas de abastecimiento arriba mencionadas. Por ejemplo, una distancia de 15 kilómetros separa a Dzibilchaltún del litoral norte de Yucatán y esto lo podríamos utilizar para argumentar que, debido a la cercanía con la costa marina, los materiales de concha llegaron a este sitio ya sea por intercambio de mercado o por todas -o algunas- de las seis formas de abastecimiento antes referidas. Además, la evidencia arqueológica de material conquiológico hallada, por ejemplo, en Cobá, Caracol, Piedras Negras, Yaxchilán, Calakmul, Tikal, también sugiere el abastecimiento muy similar a Dzibilchaltún aunque diferente a Chichén Itzá-, ya que ninguno de estos asentamientos tuvo un puerto marítimo en el litoral marino de las Tierras Bajas Mayas. Tomando en cuento lo anterior, surge la siguiente pregunta: ¿Cuál/es fue/ron la/s forma/s de abastecimiento preferidas por los sitios de las Tierras Bajas Mayas y en donde está la huella material de esa preferencia? Especulemos un poco al respecto.

Por ejemplo, el sitio de Caracol se encuentra a 130 kilómetros en línea recta del Mar Caribe, esto es, el mar que baña las islas que forman parte del arrecife de

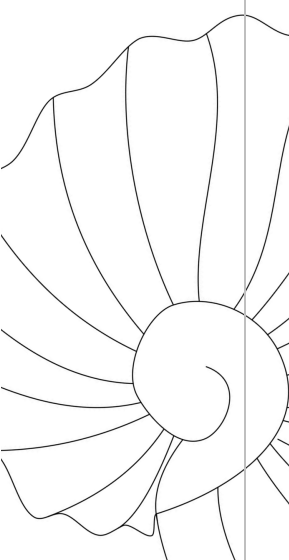




\section{Clio Arqueológica 2021, V36N2, p.149-176, COBOS;}

coral de Belice. No podemos considerar como nicho ecológico de especies de moluscos marinos el área comprendida entre tierra firme y el arrecife de coral ya que es una zona de agua dulce, resultado del enrome desfogue que realizan los numerosos ríos de Belice (ROBERTSON, 1963). Caracol también obtuvo conchas del Océano Pacífico, aunque a diferencia de los otros sitios de Tierras Bajas Mayas, su abastecimiento fue preferencialmente del Mar Caribe.

Por lo tanto, ¿cómo obtuvo Caracol su materia prima? Aquí podemos argumentar que Caracol -al igual que Kohunlich- pudo haberse abastecido de material conquiológico por medio de intercambio de mercado, o bien, haber empleado las seis formas de abastecimiento arriba mencionadas. Resulta obvio que ni el área comprendida entre tierra firme y el arrecife de coral frente a Belice, ni la Bahía de Chetumal en el sur de Quintana Roo, representaron un obstáculo para los sitios del interior para abastecerse de conchas marinas. De hecho, la evidencia reportada de Oxtankah y asentamientos vecinos ubicados a lo largo en la orilla occidental de la Bahía de Chetumal revela la preferencia de especies marinas nativas del Mar Caribe (MELGAR, 2016).

Las conchas arqueológicas halladas en Kohunlich, El Zotz, Piedras Negras, Naachtun, Calakmul y Tikal muestran claramente que la materia prima se obtuvo de tres diferentes regiones costeras que incluyeron el Golfo de México, el Mar Caribe y el Océano Pacífico (Tabla 1). A este patrón de abastecimiento se suma

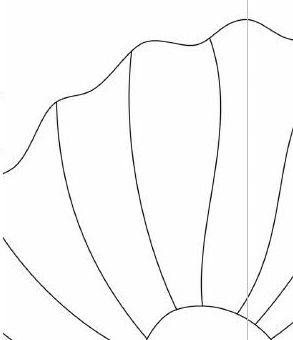




\section{Clio Arqueológica 2021, V36N2, p.149-176, COBOS;

Yaxchilán con 9 especies y 1 género nativos del Golfo de México y Mar Caribe y 6 especies del Océano Pacífico (ver Tablas 1 y 2).

Como hemos señalado líneas arriba, Caracol obtuvo su materia prima mayoritariamente del Mar Caribe en tanto que sus vecinos contemporáneos como Tikal, Calakmul, Piedras Negras y Yaxchilán tuvieron acceso a materiales tanto del Golfo de México como Mar Caribe. ¿A qué se debió la diferencia de preferencia entre Caracol y sus vecinos de las Tierras Bajas Mayas del sur? ¿Acaso la diferencia se puede atribuir a factores geográficos [Caracol está más cerca del Mar Caribe]?, o bien, ¿quizás el acceso a la materia prima nos muestra que mecanismos sociales y políticos predominaron en las preferencias en vez de un aspecto puramente económico? Estas preguntas aún esperan ser respondidas.

Conchas bivalvas Spondylus princeps y Spondylus calcifer se han encontrado completas en los numerosos entierros de la élite de sitios como Tikal, Caracol, Yaxchilán, Piedras Negras, Calakmul y se utilizaron para guardar objetos que forman parte de las ofrendas en entierros. Estos bivalvos nativos del Océano Pacífico conservaron su forma original desde que fueron recolectados en el mar y depositados en tumbas y miembros de la sociedad Maya les asignaron un alto valor social, ideológico y -posiblemente- hasta sentimental. Quizás, las conchas bivalvas Spondylus princeps y Spondylus calcifer pudieron haber sido la expresión material de regalos que intercambiaron individuos de alto estatus de manera recíproca y de forma generalizada o balanceada (SMITH, 2004). Para

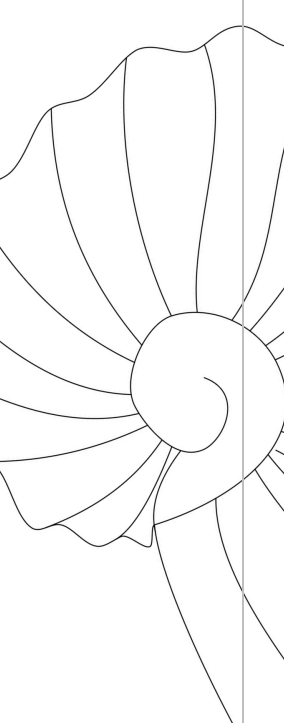


explicar este intercambio surgen dos preguntas: ¿Cuál de estas dos formas de reciprocidad fue la preferida en cada sitio? ¿Acaso las dos operaron al mismo tiempo y de forma indistinta por individuos quienes de manera consciente y deliberada fueron muy selectivos en cuanto a quien se beneficiaba de uno o el otro tipo de intercambio por reciprocidad? Con base en la evidencia disponible, no podemos -por ahora- dar respuesta a estas preguntas.

Otro aspecto a considerar del abastecimiento de Spondylus princeps y Spondylus calcifer como elementos votivos en entierros es que su presencia pudo deberse a que fueron depositados como objetos heredados que pertenecieron a la persona fallecida. De acuerdo a HUNT (2005: 293-294), cuando una persona hereda algo, esto se reconoce como una transferencia económica unidireccional e intergeneracional. Si este fue el caso, la presencia en entierros de bivalvos como Pinctada mazatlanica, Spondylus princeps y Spondylus calcifer debe ser explicada considerando el aspecto social y no tanto el económico, en otras palabras, objetos culturales cuya presencia en contextos arqueológicos se han atribuido exclusivamente a actividades de índole 'comercial' (intercambio de mercado), bien pudieron haber sido depositados en esos contextos debido a que más bien fueron heredados, regalados y, -quizás-, hasta robados. Por lo tanto, la obtención de material conquiológico también satisfizo aspectos personales que nada tuvieron que ver con el abastecimiento para la producción de objetos ornamentales.

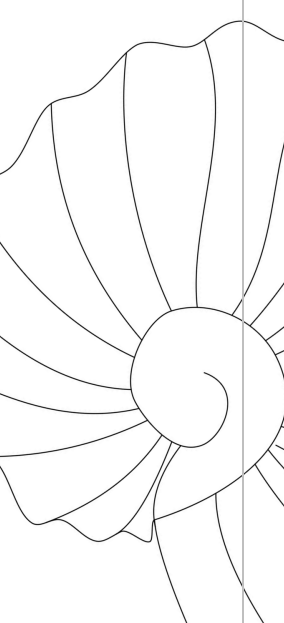




\section{Clio Arqueológica 2021, V36N2, p.149-176, COBOS; \\ DOI: 10.51359/2448-2331.2021.252353}

\section{¿CÓMO SE ORGANIZÓ LA PRODUCCIÓN DE OBJETOS DE CONCHA EN LOS SITIOS?}

Varios investigadores han reconocido que la organización de la producción ocurre - a grosso modo- entre cinco y hasta ocho formas distintas (SINOPOLI, 2003: Tabla 2.1; ver también COSTIN, 1991 y SMITH, 2004). Sin embargo, considerando la amplia literatura teórica disponible hoy día sobre producción, SINOPOLI (2003: 19, Tabla 2.1) recomienda seguir la tipología que pone especial énfasis en cinco diferentes unidades de producción. A continuación, se definen estas unidades de manera general.

La primera es la Producción Doméstica y se caracteriza por individuos que la realizan de tiempo parcial y producen productos para su autoconsumo en la unidad doméstica. La segunda es la Industria Doméstica, que involucra a individuos quienes trabajan de tiempo parcial en sus unidades domésticas y producen objetos que intercambian para ser consumidos en otras unidades domésticas. En tercer lugar está la Industria de Taller -también denominada como Talleres Nucleados o Especialización Comunal-, donde hay trabajadores de tiempo completo quienes laboran en talleres especializados con el fin de generar una sobreproducción de objetos. La Industria de la Comunidad -nombrada también como Especialización Comunitaria o Especialización de la Comunidades la cuarta forma y se caracteriza por la presencia de artesanos especialistas que residen en sus comunidades y producen objetos para una distribución regional. La

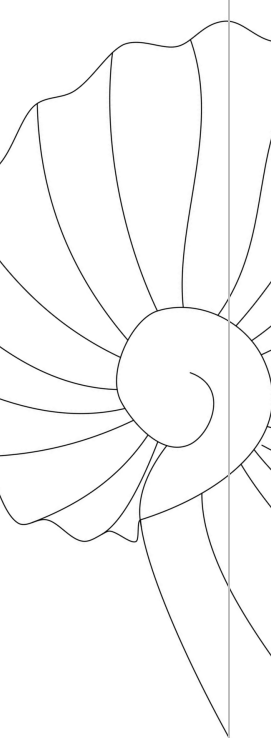


quinta y última forma se denomina Producción en Talleres y, en esta forma, los talleres se encuentran aislados físicamente de unidades domésticas, exhiben un alto nivel de especialización y predomina una estandarización en la producción de objetos (SINOPOLI, 2003: 17-20; Tabla 2.1).

Tomando en consideración las cinco unidades de producción definidas en el párrafo inmediato superior, nos preguntamos: ¿en dónde está la evidencia arqueológica de cada una de ellas en el área Maya? Una revisión de los datos arqueológicos sugiere -por ahora- que la manufactura y obtención de objetos de concha en las Tierras Bajas Mayas se realizó de dos maneras: por Producción Doméstíca y por Industria Doméstica. En cuanto a la Industria de Taller, Industria de la Comunidad y Producción en Talleres de concha, estas continúan siendo evasivas en el registro arqueológico del área Maya ya que aún no se reportan talleres en los que hubo una sobreproducción de esos objetos.

Un primer ejemplo de Producción Doméstica se reporta en el Grupo Mosquito de Caracol. Este conjunto doméstico se localiza a 1.1 kilómetros al sureste del centro del sitio. Un total de 1,815 fragmentos no trabajados, 9 anillos, 5 botones, 2 discos y 2 piezas trabajadas integran la colección de piezas del grupo. Con la excepción de dos discos que se obtuvieron de la especie bivalva Spondylus americanus, los otros 1,813 objetos se obtuvieron exclusivamente de Strombus gigas (COBOS, 1994). Las numerosas piezas fragmentadas de Strombus gigas halladas en el Grupo Mosquito se encontraron asociadas con herramientas de pedernal que 


\section{Clio Arqueológica 2021, V36N2, p.149-176, COBOS;

muestran un patrón de estandarización repetido en sus formas que incluyen perforadores, cuñas, raspadores y lascas bifaciales (POPE, 1994).

Un segłndo ejemplo de Producción Doméstica se reporta de la Plataforma 8M001 (FN-129) del sitio Xuenkal en Yucatán (ALONSO et al., 2009; ALONSO, 2013). La muestra de material de concha está representada por 408 objetos que incluyen ornamentos y elementos utilitarios. Las especies marinas más aprovechadas para la manufactura de los objetos de concha en Xuenkal incluyeron a los caracoles Strombus costatus, Strombus gigas, Pleuroploca gigantea y Turbinella angulata, todos ellos nativos de la cercana costa norte de Yucatán. Con base en el análisis del material, se destaca que la producción de los objetos de concha fue de mediana intensidad para obtener una gama limitada de objetos, en vez de una producción intensiva. La producción de los objetos de concha en Xuenkal sirvió tanto para el autoconsumo doméstico como -aparentemente- para el intercambio interno en el sitio (ALONSO et al., 2009; ALONSO, 2013).

El material conquiológico de Xuenkal no muestra todos los pasos de la secuencia en su manufactura y esta falta de evidencia le sugirió a ALONSO (2013) dos explicaciones. La primera es que los artesanos de Xuenkal:

"solamente tuvieron un grado limitado de especialización para la producción de objetos simples" (ALONSO, 2013: 299).

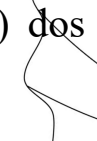




\section{Clio Arqueológica 2021, V36N2, p.149-176, COBOS;

En la segunda explicación se argumenta que los artesanos se dedicaron a la manufactura de unos cuantos objetos utilitarios pequeños, así como de pocos ornamentos (ALONSO, 2013: 299). Una tercera explicación podría ser que los ornamentos y objetos utilitarios llegaron manufacturados a Xuenkal desde Chichén Itzá ya que el sitio funcionó como estación terrestre de paso entre Isla Cerritos y Chichén Itzá durante los siglos X y XI (ARDREN Y LOWRY, 2011; COBOS, 2014). De hecho, la Plataforma 8M-001 (FN-129) presenta una ocupación tardía que corresponde al período Clásico Terminal (800 dC. - 1100 dC.) y la hace contemporánea tanto a Chichén Itzá como a Isla Cerritos (ARDREN Y LOWRY, 2011; ALONSO, 2013; COBOS, 2014).

El segundo nivel de producción que reconocemos en este trabajo se denomina Industria Doméstica y los ejemplos que utilizamos proceden de Tikal, Copán, Calakmul y Aguateca. En el primero de estos sitios la evidencia arqueológica sugiere que artesanos especialistas vivieron y trabajaron de tiempo parcial en los numerosos grupos de estructuras menores que se encuentran en el área central de Tikal y muy cercanos a los grandes conjuntos monumentales. Estos artesanos produjeron objetos de concha que fueron consumidos tanto por el grueso de la población como por miembros de la elite (MOHOLY-NAGY, 1995: 9). De hecho, MOHOLY-NAGY (1995: 7) reconoció la existencia de dos agrupamientos de materiales conquiológicos asociados con toda la ocupación de Tikal. El primero se denomina 'Conjunto de Rango Superior' que se asocia exclusivamente con la

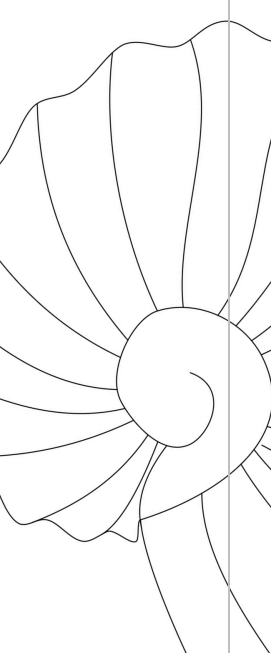




\section{Clio Arqueológica 2021, V36N2, p.149-176, COBOS;

élite; el segundo es el 'Conjunto de Rango Inferior' asociado con una elite menor, o bien, con personas con riqueza, aunque no pertenecientes a la elite.

Los objetos de concha del 'Conjunto de Rango Superior' se encontraron asociados tanto con una elaborada arquitectura como con depósitos muy exclusivos, particularmente entierros y escondites especiales resultado de las actividades efectuadas por miembros de la élite (MOHOLY-NAGY, 1995: 7). Un rasgo que destaca de los 'Conjuntos de Rango Superior' es el énfasis en el uso de conchas del género Spondylus (MOHOLY-NAGY, 1995: 7). Los objetos de concha asociados con el 'Conjunto de Rango Inferior' se recobraron de contextos generales excavados y son mayoritariamente ornamentales (MOHOLY-NAGY, 1995: 8).

En Copán, (Honduras), los ornamentos de concha fueron el resultado de una producción de bajo volumen realizada por artesanos tanto en el patio Oeste del Grupo Principal -enfrente de la Estructura 10L-26- como en el Grupo Arquitectónico 9N-8 (ver especialmente Estructura 110B), un conjunto residencial asociado con la elite y ubicado en el Grupo Sepulturas al noreste del centro de Copán (WEBSTER et al., 1993; AOYAMA, 1995). Estos artesanos fueron especialistas de medio tiempo quienes dependieron y estaban al servicio tanto de miembros de la nobleza como también de los gobernantes de Copán. Además, se sugiere que una parte de esos artesanos de medio tiempo también formaron parte de la elite (WEBSTER et al., 1993: 286; AOYAMA, 1995: 140-141).

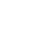




\section{Clio Arqueológica 2021, V36N2, p.149-176, COBOS;

En Calakmul se reporta la existencia de:

"artesanos especializados pertenecientes a una clase media baja" (DOMÍNGUEZy FOLAN, 1999: 712).

Además, a estos artesanos de clase media baja se suman artesanos pertenecientes a ta elite quiènes vivían en los cuartos de la parte inferior de la Estructura II, una elaborada construcción tipo palacio. De acuerdo a DOMÍNGUEZ y FOLAN (1999: 713), siete cuartos de la fachada norte de la Estructura II evidenciaron los restos de trabajo en la manufactura de piezas de concha.

Los artesanos especializados de Calakmul se encargaron de manufacturar numerosos objetos ornamentales, utilitarios y votivos de concha. Además, hay evidencia de materiales de concha no trabajados y piezas semi-trabajadas asociadas con la Estructura II, estructura en la cual se realizaba la producción de objetos de concha. Una vez concluida la manufactura de las piezas en los cuartos de la parte inferior de la Estructura II, los objetos fueron enviados a miembros de la elite para su uso quienes residían -por ejemplo- en las Estructuras IIB y III también de tipo palacio.

De Aguateca procede otro ejemplo de Industria Doméstica y el estudio del material conquiológico sugiere que un sector de los residentes de Aguateca estaban involucrados en la manufactura de objetos de concha y hueso de animales (EMERY Y AOYAMA, 2007). La evidencia arqueológica de restos de concha fue

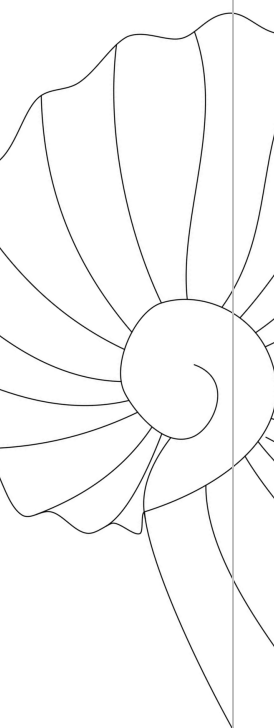




\section{Clio Arqueológica 2021, V36N2, p.149-176, COBOS;

hallada en las estructuras M8-4, M8-8 (parte posterior al sur de la construcción), M8-10 (basurero al sur de la estructura) y M8-13.

Las conclusiones del estudio faunístico y lítico realizado con el material arqueológico de Aguateca, le sugirieron a EMORY y AOYAMA (2007: 84) lo siguiente. Primero, la producción de objetos de concha revela algunos de los pasos del proceso de manufactura, con excepción del primer paso asociado con la secuencia de reducción de las piezas. Segundo, sugirieron que miembros de la elite Maya residentes de Aguateca:

"were involved in the production of bone artifacts, hide and other textiles, and shell adornments. (EMERY y AOYAMA, 2007: 84).

\section{CONCLUSIÓN}

Cuando nos referimos a la producción de objetos de concha utilizando una perspectiva económica resulta evidente la complejidad de este proceso, especialmente cuando consideramos tanto la manera en la cual se obtuvo la materia prima y cómo pudo haberse organizado la producción a nivel de sitio. En relación al abastecimiento de conchas marítimas, los numerosos sitios ubicados en el interior del área Maya pudieron haber obtenido la materia prima de las siguientes maneras: por intercambio de mercado ('comercio'); por encargo ya que la materia prima era enviada a los sitios del interior; por artesanos que fueron hasta el litoral marino a obtener esa materia prima; por haberla obtenido en un

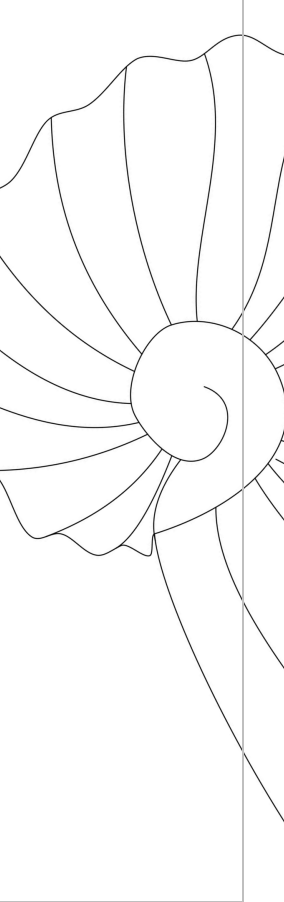




\section{Clio Arqueológica 2021, V36N2, p.149-176, COBOS;

punto intermedio entre la comunidad y la costa marina; por tributo o impuesto; por intercambio de regalos manifestando ya sea reciprocidad balanceada y/o generalizada; por el hurto o robo de la materia prima. Cabe indicar que aún estamos lejos de poder determinar cual/es de esta/s formas de abastecimiento fueron las preferidas de los hábiles artesanos encargados de la producción de piezas utilitarias y ornamentales de concha.

Con base en los datos derivados de los distintos estudios sobre conchas arqueológicas en el área Maya, resulta evidente que la producción de objetos de concha se realizó en unidades domésticas asociadas con miembros de la elite y por gente común. Esta producción satisfizo el autoconsumo en esas unidades domésticas (Producción Doméstica), así como su consumo en otras unidades domésticas (Industria Doméstica).

Las unidades domésticas del común denominador de la población parecen haber jugado un papel primordial en la producción de ornamentos, objetos utilitarios y votivos para satisfacer su autoconsumo. También las unidades domésticas asociadas con la elite elaboraron objetos de concha para satisfacer una afanosa demanda. Como hemos visto, los artesanos trabajando para individuos de alto estatus fueron -aparentemente- expertos orfebres de clase media que trabajaban de tiempo parcial para la elite, aunque también existieron hábiles artesanos que parecen haber pertenecido a la elite. 


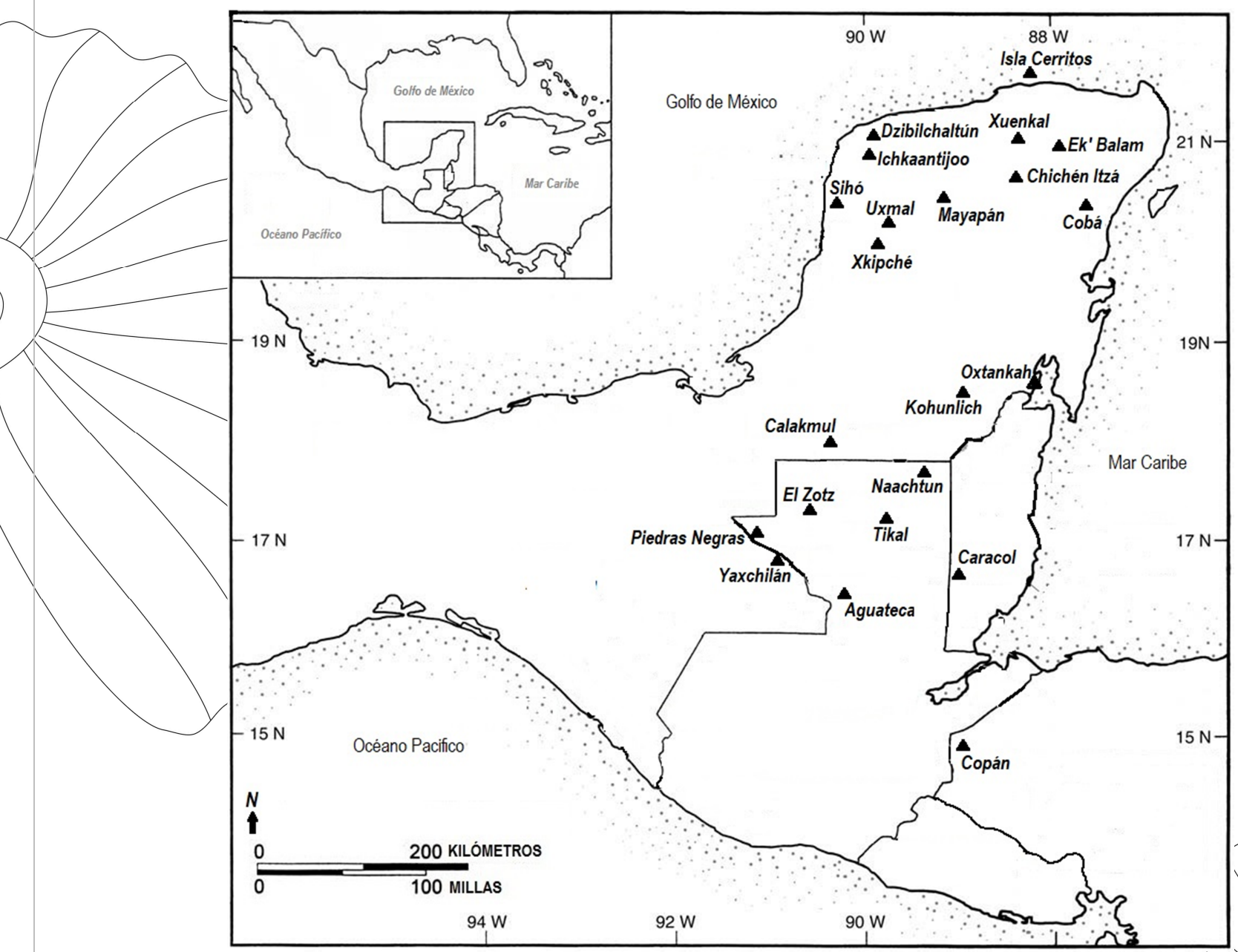

Figura 1. Mapa con la ubicación de los sitios mencionados en el texto, (dibujó R. Cobos).

El presente trabajo ha seguido una perspectiva netamente económica para analizar la producción de objetos de concha en sitios de las Tierras Bajas del área Maya 


\section{Clio Arqueológica 2021, V36N2, p.149-176, COBOS;

durante los períodos Clásico Tardío y Clásico Terminal. Este ejercicio académico revela que empezamos a responder muy contadas preguntas sobre producción con ese tipo de materiales; sin embargo, aún quedan numerosos cuestionamientos sobre producción y esto representa un verdadero reto de estudio para poder eomprender quienes, cómo y finalidad de la producción de las distintas piezas de concha que hoy día representan nuestro tema de investigación.

\begin{tabular}{|c|c|c|c|c|}
\hline $\begin{array}{l}\text { Sitios del interior } \\
\text { de las tierras } \\
\text { bajas }\end{array}$ & $\begin{array}{c}\text { Número total } \\
\text { de } \\
\text { piezas } \\
\text { reportadas }\end{array}$ & $\begin{array}{c}\text { Procedencia: Golfo } \\
\text { de México y/o Mar } \\
\text { Caribe }\end{array}$ & $\begin{array}{c}\text { Procedencia: } \\
\text { Océano } \\
\text { Pacífico }\end{array}$ & Agua dulce \\
\hline \multicolumn{5}{|l|}{$\begin{array}{c}\text { Tierras Bajas del } \\
\text { Norte }\end{array}$} \\
\hline Chichén Itzá & 562 & 29 especies & 0 & $\begin{array}{l}2 \text { especies y } 1 \\
\text { género }\end{array}$ \\
\hline Cobá & 292 & 5 especies & 5 especies & 1 especie \\
\hline Dzibilchaltún & 2,376 & 71 especies & 0 & 2 especies \\
\hline Ek' Balam & 7,169 & $\begin{array}{l}22 \text { especies y } 2 \\
\text { géneros }\end{array}$ & 2 especies & 1 especie \\
\hline Ichkaantijoo & 276 & $\begin{array}{l}24 \text { especies y } 7 \\
\text { géneros }\end{array}$ & $\begin{array}{c}1 \text { especie y } 1 \\
\text { género }\end{array}$ & 1 especie \\
\hline Mayapán & 337 & $\begin{array}{l}32 \text { especies y } 1 \\
\text { género }\end{array}$ & 0 & $\begin{array}{l}1 \text { especie y } 2 \\
\text { géneros }\end{array}$ \\
\hline $\begin{array}{c}\text { Oxtankah y sitios } \\
\text { cercanos }\end{array}$ & 2,185 & $\begin{array}{l}63 \text { especies y } 4 \\
\text { géneros }\end{array}$ & 5 especies & $\begin{array}{c}2 \text { especies y } 2 \\
\text { géneros }\end{array}$ \\
\hline Sihó & 418 & 35 especies y 2 & 0 & 1 especie 1 \\
\hline
\end{tabular}




\section{Clio Arqueológica 2021, V36N2, p.149-176, COBOS;

La información se obtuvo de las siguientes fuentes: Calakmul (MELGAR TÍSOC y DOMÍNGUEZ CARRASCO, 2017), Caracol (COBOS, 1994), Chichén Itzá (COBOS, 1989), Cobá (CASTILlO VELASCO, 2020), Dzibilchaltún (ANDREWS IV, 1969), Ek' Balam (HERNÁNDEZ et al., 2014), El Zotz (GUTIÉRREZ CASTILLO, 2015), Ichkaantijoo (GÓMEZ COBÁ y PANTOJA DÍAZ, 2018), Kohunlich (REYES ESPINOSA, 2012, 2020), Mayapán (COBOS y PERAZA LOPE, 2006), Naachtun (COTOM NIMATUJ, 2019), Oxtankah (MELGAR TÍSOC, 2016), Piedras Negras (COE, 1959), Sihó (COBOS, 2010), Tikal (MOHOLY-NAGY, 1963, 1995), Uxmal (COBOS, 2010), Xkipché (COBOS, 2010), Xuenkal (ALONSO et al. 2009; ALONSO 2013), Yaxchilán (VELÁZQUEZ CASTRO et al., 2014; COBOS en este trabajo este trabajo).

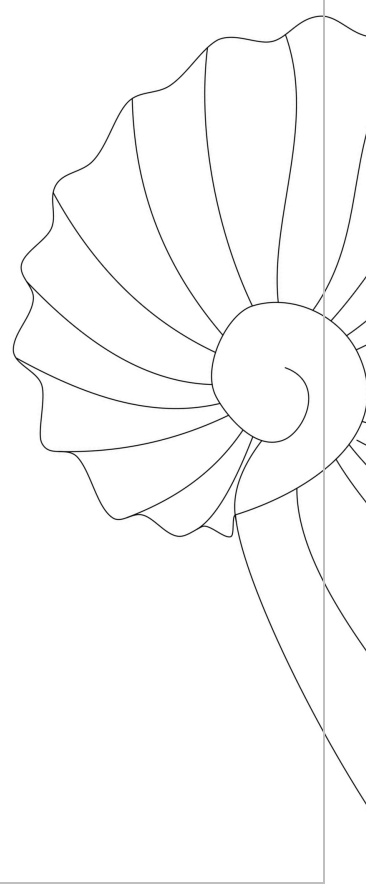




\begin{tabular}{|c|c|c|c|c|}
\hline $\begin{array}{l}\text { GASTERÓPODO } \\
\text { Especies }\end{array}$ & & & & \\
\hline $\begin{array}{c}\text { Golfo de México y Mar } \\
\text { Caribe }\end{array}$ & $\begin{array}{l}\text { Fragmento no } \\
\text { trabajado }\end{array}$ & $\begin{array}{l}\text { Fragmento } \\
\text { trabajado }\end{array}$ & Entero trabajado & Total \\
\hline Strombus sp. & 0 & 36 & 0 & 36 \\
\hline Oliva reticularis & 0 & 0 & 6 & 6 \\
\hline Trivia (Pusula) pullata & 1 & 0 & 4 & 5 \\
\hline No identificables & 0 & 7 & 0 & 7 \\
\hline \multicolumn{5}{|l|}{ PELECIPODO / Especies } \\
\hline $\begin{array}{c}\text { Golfo de México y Mar } \\
\text { Caribe }\end{array}$ & $\begin{array}{l}\text { Fragmento no } \\
\text { trabajado }\end{array}$ & $\begin{array}{l}\text { Fragmento } \\
\text { trabajado }\end{array}$ & Entero trabajado & Total \\
\hline Spondylus americanus & 1 & 607 & 8 & 616 \\
\hline \multicolumn{5}{|l|}{ Océano Pacífico } \\
\hline Spondylus calcifer & 0 & 0 & 2 & 2 \\
\hline Spondylus princeps & 0 & 0 & 1 & 1 \\
\hline Pinctada mazatlanica & 0 & 0 & 2 & 2 \\
\hline \multicolumn{5}{|l|}{$\begin{array}{c}\text { Ríos de agua dulce y } \\
\text { aguadas de Tabasco y/o } \\
\text { Guatemala }\end{array}$} \\
\hline $\begin{array}{c}\text { Nephronaias/Psoronaias } \\
s p .\end{array}$ & 1 & 8 & 0 & 9 \\
\hline Total & 3 & 658 & 23 & 684 \\
\hline
\end{tabular}

Tabla 2. Yaxchilán: especies de moluscos arqueológicos hallados en las Tumbas 1 a 6 del sitio. 


\section{Clio Arqueológica 2021, V36N2, p.149-176, COBOS; DOI: 10.51359/2448-2331.2021.252353}

\section{REFERENCIAS BIBLIOGRÁFICAS}

ABBOTT, R.T. 1996. A guide to field identification seashells of North America. New York: St. Martin Press.

ALONSO, A. 2013. Economic strategies of Terminal Classic households in the northern Maya lowlands: Multicrafting and economic diversification of a mid-elite residential compound at Xuenkal, Yucatan. Tesis de Doctorado en Antropología. Alberta: University of Calgary.

ALONSQ, A., T.K. MANAHAN, y T. ARDREN. 2009. "La producción de bienes de concha y caracol y la economía política de Xuenkal". Encuentro internacional. Los investigadores de la cultura maya 2008, vol. 17, 265-283.

ANDREWS, E.W., IV. 1969. The archaeological use and distribution of mollusca in the maya lowlands. Middle American Research Institute, Pub. 34. New Orleans: Tulane University.

AOYAMA, K. 1995. "Microwear analysis in the southeast maya lowlands: Two case studies at Copan, Honduras". Latin American Antiquity vol. 6 (2), 129-144.

ARDREN, T. y J. LOWRY. 2011. "The travels of maya merchants in the ninth and tenth centuries AD: Investigations at Xuenkal and the Greater Cupul Province, Yucatan, Mexico". World Archaeology vol 43 (3): 428-443.

BERDAN, F.F., M.A. MASSON, J. GASCO, y M.E. SMITH. 2003. “An international economy". En M.E. Smith y F.F. Berdan (eds.). The Postclassic Mesoamerican World. Salt Lake City: University of Utah Press, 96-108.

CASTILLO, E.A. 2020. La producción de objetos de concha recuperados em las ofrendas de Cobá, Quintana Roo. Estudios de cultura maya vol. 55, 89-119.

COBOS, R. 1989. Shelling in: Marine mollusca at Chichén Itzá. En H. McKillop y P.F. Healy (eds.). Coastal Maya Trade. Trent University, Occasional Papers in Anthropology No. 8. Ontario: Peterborough, 49-58.

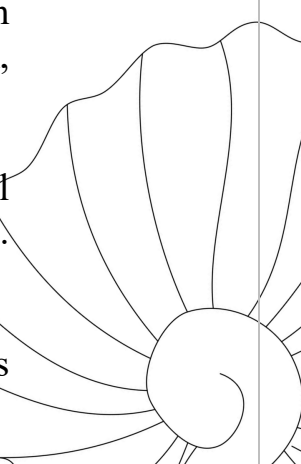




\section{Clio Arqueológica 2021, V36N2, p.149-176, COBOS; DOI: 10.51359/2448-2331.2021.252353}

COBOS, R. 1994. Preliminary report on the archaeological mollusca and shell ornaments of Caracol, Belize. En D.Z. Chase y A.F. Chase (eds.). Studies in the Archaeology of Caracol, Belize, Monograph 7. San Francisco: Pre-Columbian Art Research Institute, $139-147$.

COBOS, R. 2010. "Conchas arqueológicas en el occidente de Yucatán: patrones de abastecimiento durante el Clásico Tardío y el Clásico Terminal". En L. Suárez Díez y A. Velázquez Castro (coordinadores). Ecos del pasado: los moluscos arqueológicos de México. Colección Científica 572, Serie Etnohistoria. México: Instituto Nacional de Antropología e Historia, 47-63.

COBOS, R. 2013. Intercambio de mercado en el área Maya. Arqueología Mexicana 122, $54-61$.

COBOS, R. 2014. Conchas marinas en Chichén Itzá: abastecimiento y uso durante el Clásico Términal. En C.M. Götz, J. Rivas Romero, J.M. Cárdenas Cervantes, H. Hernández Álvarez, M. Zimmermann y C. Romero Novelo (compiladores). Memorias del Congreso Internacional Culturas Americanas y su Ambiente: Perspectivas desde la Zooarqueología, Paleobotánica y Etnobiología. Mérida: Ediciones de la Universidad Autónoma de Yucatán, 256-264.

COBOS, R. y C. PERAZA LOPE. 2006. Mayapán y sus conchas arqueológicas: implicaciones para reconstruir antiguas rutas de abastecimiento peninsular durante el Posclásico. Los investigadores de la cultura maya 14, tomo II, 544-552.

COE, W.R. 1959. Piedras Negras Archaeology: Artifacts, Caches and Burials. Museum Monographs No. 4, The University Museum. Philadelphia: University of Pennsylvania.

COSTIN, C.L. 1991. Craft specialization: Issues in defining, documenting, and explaining the organization of production. En M.B. Schiffer, (ed.). Archaeological method and theory. Tucson: The University of Arizona Press, 1-56.

COTOM, J.A. 2019. ¿Y cómo lo hicieron? La manufactura de los objetos de concha del Clásico Tardío-Terminal (c.730.950/1000 d.C.) en Naachtun, Guatemala. Tesis de Maestría en Arqueología. La Piedad: El Colegio de Michoacán, A.C.

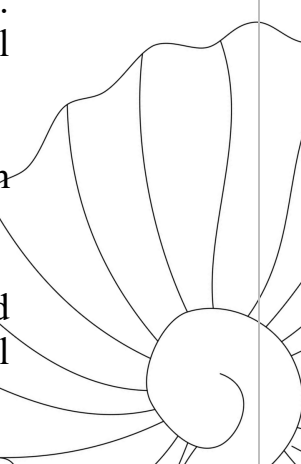




\section{Clio Arqueológica 2021, V36N2, p.149-176, COBOS; DOI: 10.51359/2448-2331.2021.252353}

DOMÍNGUEZ, M.R. y W.J. FOLAN. 1999. Hilado, confección y lapidación: los quehaceres cotidianos de los artesanos de Calakmul, Campeche, México. En J.P. Laporte, H.L. Escobedo y A.C. Monzón de Suasnávar (eds.). XII Simposio de Investigaciones Arqueológicas en Guatemala, 1998, Guatemala: Ministerio de Cultura y Deportes, Instituto de Antropología e Historia, Asociación Tikal, 711-730.

EMERY, K. y K. AOYAMA. 2007. Bone, shell, and lithic evidence for crafting in elite Maya households at Aguateca, Guatemala. Ancient Mesoamerica vol. 18, 69-89.

FEINMAN, G.M. y C.P. GARRATY. 2010. Preindustrial markets and marketing: Archaeological perspectives. Annual Review of Anthropology vol. 39, 167-191.

FEINMAN, G.M. y L.M. NICHOLAS. 1993. Shell-ornament production in Ejutla. Implications for highland-coastal interaction in ancient Oaxaca. Ancient Mesoamerica vol. 4, 103-119.

FEINMAN, G.M. y L.M. NICHOLAS. 1995. Household craft specialization and shell ornament manufacture in Ejutla, México. Expedition vol. 37, 14-24.

FEINMAN, G.M. y L.M. NICHOLAS. 2004. Unraveling the prehispanic highland Mesoamerican economy: Production, exchange, and consumption in the Classic-period valley of Oaxaca. En G.M. FEINMAN y L.M. NICHOLAS (eds.). Archaeological perspective on political economies. Salt Lake City: University of Utah Press, 167-188.

GÓMEZ. M.J. y L. PANTOJA. 2018. Del mar a la tierra. Aprovechamiento de los recursos malacofaunísticos en sitios de la región de Ichkaantijoo, Yucatán. En M.R. Domínguez Carrasco, M.J. Gallegos Gómora, R. Armijo Torres y M.E. León Méndez (eds.). Los investigadores de la cultura maya, gastronomía en la cultura maya: usos cotidianos. Campeche: Universidad Autónoma de Campeche, 91-108.

HERNÁNDEZ, C.A., L. VARGAS y V. CASTILLO. 2014. Los objetos de concha de Ukit Kan Lek Tok', rey de Ek' Balam”. Los Investigadores de la Cultura Maya 22, tomo II, 221-236.

HIRTH, K.G. 2016. The aztec economic world, merchants and markets in ancient Mesoamerica. Cambridge: Cambridge University Press.

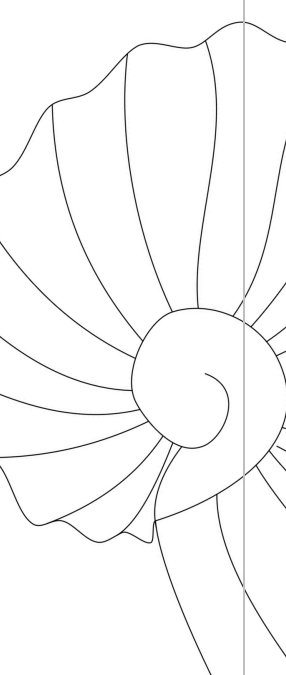




\section{Clio Arqueológica 2021, V36N2, p.149-176, COBOS;

HUNT, R.C. 2005. One-way economic transfers. En J.G. Carrier (ed.). A Handbook of Economic Anthropology. Cheltenham, UK and Northhampton, MA: Edward Elgar Publishing, Inc., 290-301.

JUÁREZ, D. 2012. La pequeña acrópolis de Yaxchilán en el contexto de la arquitectura palaciega. En L.S. Lowe y M.E. Pye (eds.). Arqueología reciente de Chiapas: contribuciones del encuentro celebrado en el $60^{\circ}$ aniversario de la Fundación Arqueologica Nuevo Mundo. Papers of the New World Archaeological Foundation 72. Provo: Brigham Young University, 327-339.

MANZANILLA, L., X. BOKHIMI, D. TENORIO, M. JIMÉNEZ-REYES, E. ROSALES, C. MARTÍNEZ, y M. WINTER. 2017. Procedencia de la mica de Teotihuacan: control de los recursos suntuarios foráneos por las élites gobernantes. Anales de Antropología 51, 23-38.

MELGAR, E.R. 2016. Shell materials from Oxtankah, Quintana Roo. En D.S. Walker (ed.). Perspectives on the Ancient Maya of Chetumal Bay. Gainesville: University Press of Florida, 217-232.

MELGAR, E.R. y M.R. DOMÍNGUEZ. 2014. Los artesanos de concha y la élite de Calakmul: los objetos elaborados y sus técnicas de manufactura. Encuentro Internacional de los Investigadores de la Cultura Maya 2013, vol. 22, 203-219.

MELGAR, E.R. y M.R. DOMÍNGUEZ. 2017. Los moluscos de Calakmul: procedencia, rutas de obtención y manufactura. En M.R. Domínguez Carrasco, M.J. Gallegos Gómora, R. Armijo Torres y M.E. León Méndez (eds.). Los Investigadores de la Cultura Maya, el Comercio y otros Temas, 111-123.

MOHOLY-NAGY, H. 1963. Shells and other marine material from Tikal. Estudios de Cultura Maya vol. III, 65-83.

MOHOLY-NAGY, H. 1995. "Shells and society at Tikal, Guatemala". Expedition vol. 37: 3-13.

POPE, C.L. 1994. Preliminary analysis of small chert tools and related debitage at Caracol, Belize". En D.Z. Chase y A.F. Chase (eds.). Studies in the Archaeology of Caracol, Belize, Monograph 7. San Francisco: Pre-Columbian Art Research Institute, $148-156$. 


\section{Clio Arqueológica 2021, V36N2, p.149-176, COBOS; DOI: 10.51359/2448-2331.2021.252353}

RENFREW, C. 1975. "Trade as action at a distance: Questions of integration and communication". En J.A. Sabloff y C.C. Lamberg-Karlovsky (eds.). Ancient Civilizations and Trade, Albuquerque: University of New Mexico Press, 3-59.

REYES, A. 2012. La producción especializada de objetos de concha en Kohunlich, Quintana Roo. Tesis de Licenciatura. México: Escuela Nacional de Antropología e Historia.

REYES, A. 2018. "Producción de objetos manufacturados en Strombus gigas, de Kohunlich, Quintana Roo". En E.R. Melgar Tísoc y L.R. Manzanilla Naim (coordinadores). Arqueología de la Producción, Colección Arqueología, Serie Logos.

Méxieo: Secretaría de Cultura, Instituto Nacional de Antropología e Historia, Universidad Nacional Autónoma de México - Instituto de Investigaciones Antropológicas, 123-161.

ROBERTSON, R. 1963. "The mollusks of British Honduras". En K.F. Wantland y W.C. Pusey III (eds.). Belize shelf-carbonate sediments, clastic sediments, and ecology. Oklahoma: The American Association of Petroleum Geologists, Appendix: 42-53.

SINOPOLI, C.M. 2003. The political economy of craft production. Cambridge: Cambridge University Press.

SMITH, M.E. 2004. "The archaeology of ancient state economies". Annual Review of Anthropology vol. 33: 73-102.

VELÁZQUEZ, A. y L.S. LOWE (eds.). 2007. Los moluscos Aarqueológicos. Una visión del mundo maya. Cuadernos del Centro de Estudios Mayas. México: Universidad Nacional Autónoma de México.

VELÁZQUEZ, A., D. JUÁREZ, B. ZÚÑIGA, N. VALENTÍN. 2013. "La producción de artefactos de concha en la Pequeña Acrópolis de Yaxchilán, Chiapas". En A. Velázquez Castro y L.S. Lowe (eds.). Técnicas analíticas aplicadas a la caracterización y producción de materiales arqueológicos en el área maya. México: Universidad Nacional Autónoma de México, Instituto de Investigaciones Filológicas, 31-65. 
VOKES, H.E. y E.H. VOKES. 1983. Distribution of shallow-water marine mollusca, Yucatan peninsula, Mexico. Mesoamerican Ecology Institute, Monograph 1. New Orleans: Middle American Research Institute, Tulane University.

WEBSTER, D.L., S.T. EVANS y W.T. SANDERS. 1993. Out of the Past. California: Mayfiete, Mountainview.

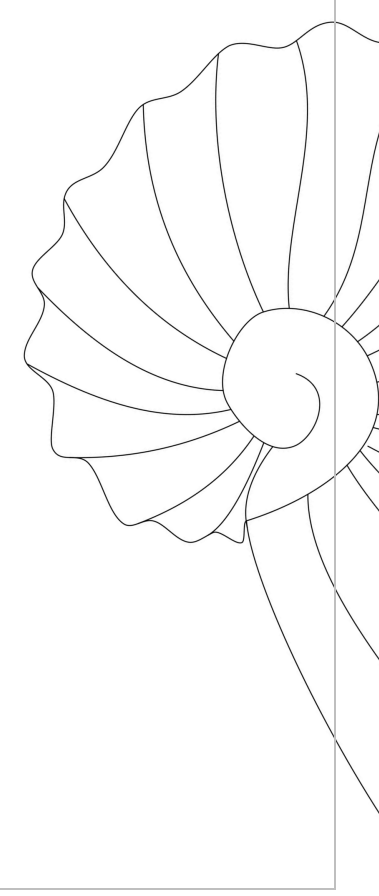

